\title{
Studies of small-scale plasma inhomogeneities in the cusp ionosphere using sounding rocket data
}

Alexander A. Chernyshov, Andres Spicher, Askar A. llyasov, Wojciech J. Miloch, Lasse B. N. Clausen, Yoshifumi Saito, Yaqi Jin, and Jøran I. Moen

Citation: Physics of Plasmas 25, 042902 (2018); doi: 10.1063/1.5026281

View online: https://doi.org/10.1063/1.5026281

View Table of Contents: http://aip.scitation.org/toc/php/25/4

Published by the American Institute of Physics

\section{Articles you may be interested in}

Electron acoustic nonlinear structures in planetary magnetospheres

Physics of Plasmas 25, 042303 (2018); 10.1063/1.5026186

A study of the asymmetrical distribution of solar activity features on solar and plasma parameters (1967-2016)

Physics of Plasmas 25, 042901 (2018); 10.1063/1.5018058

Drift turbulence, particle transport, and anomalous dissipation at the reconnecting magnetopause

Physics of Plasmas 25, 062103 (2018); 10.1063/1.5027086

Scattering by the broadband electrostatic turbulence in the space plasma

Physics of Plasmas 25, 072903 (2018); 10.1063/1.5039687

Vlasov dynamics of periodically driven systems

Physics of Plasmas 25, 042302 (2018); 10.1063/1.5026159

Bifurcations in the theory of current transfer to cathodes of DC discharges and observations of transitions between different modes

Physics of Plasmas 25, 042307 (2018); 10.1063/1.5024383

\section{PHYSICS TODAY}

MANAGER'S GUIDE

Accelerate R\&D with Multiphysics Simulation
READ NOW

PRESENTED BY

○○ $\subset$ MSOL 


\title{
Studies of small-scale plasma inhomogeneities in the cusp ionosphere using sounding rocket data
}

\author{
Alexander A. Chernyshov, ${ }^{1, a)}$ Andres Spicher, ${ }^{2, b)}$ Askar A. Ilyasov, ${ }^{1, c)}$ Wojciech J. Miloch, ${ }^{2, d)}$ \\ Lasse B. N. Clausen, ${ }^{2, e)}$ Yoshifumi Saito, ${ }^{3, f)}$ Yaqi Jin, ${ }^{2, g)}$ and Jøran I. Moen ${ }^{2, h)}$ \\ ${ }^{1}$ Space Research Institute of the Russian Academy of Science, Moscow 117997, Russia \\ ${ }^{2}$ Department of Physics, University of Oslo, Box 1048 Blindern, N-0316 Oslo, Norway \\ ${ }^{3}$ Institute of Space and Astronautical Science, Japan Aerospace Exploration Agency, Sagamihara, Kanagawa, \\ Japan
}

(Received 17 February 2018; accepted 13 March 2018; published online 3 April 2018)

\begin{abstract}
Microprocesses associated with plasma inhomogeneities are studied on the basis of data from the Investigation of Cusp Irregularities (ICI-3) sounding rocket. The ICI-3 rocket is devoted to investigating a reverse flow event in the cusp $F$ region ionosphere. By numerical stability analysis, it is demonstrated that inhomogeneous-energy-density-driven (IEDD) instability can be a mechanism for the excitation of small-scale plasma inhomogeneities. The Local Intermittency Measure (LIM) method also applied the rocket data to analyze irregular structures of the electric field during rocket flight in the cusp. A qualitative agreement between high values of the growth rates of the IEDD instability and the regions with enhanced LIM is observed. This suggests that IEDD instability is connected to turbulent non-Gaussian processes. Published by AIP Publishing.
\end{abstract}

https://doi.org/10.1063/1.5026281

\section{INTRODUCTION}

Plasma irregularities and turbulence with scale-size ranging from hundreds of kilometers to a few meters are common phenomena in the high latitude $F$ region ionosphere (e.g., Refs. 1-4). The density irregularities are regarded as challenging space weather issues, as they are known to disturb radio communications and create scintillations that can affect Global Navigation Satellite System (GNSS) signals (e.g., Refs. 4-7). Understanding and characterizing different mechanisms leading to plasma irregularities in the highlatitude ionosphere is thus crucial for future space weather forecast models (e.g., Ref. 4). The possible source mechanisms for plasma irregularities are broad, including both macro- and micro-instability mechanisms, and comprise, among others, the Gradient-Drift instability (GDI), which occurs on density gradients, and which is often mentioned in relation to polar cap patches (e.g., Refs. 1, 2, and 8), electrostatic ion cyclotron (EIC) and ion acoustic (IA) waves which can be driven unstable by field-aligned particles velocity, ${ }^{9}$ or flow shear instability mechanisms arising due to inhomogeneous electric fields (e.g., Refs. 10-13).

Inhomogeneous electric fields are frequent in the highlatitude ionosphere ${ }^{10}$ and can drive the plasma unstable through the Kelvin-Helmholtz instability (KHI). ${ }^{11,14}$ It is noteworthy that even a small shear can initiate the development of the KHI. ${ }^{15}$ Due to its large-scale character, the KHI alone

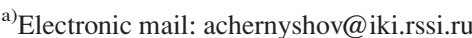

b)Electronic mail: andres.spicher@gmail.com

${ }^{c}$ Electronic mail: asjosik@mail.ru

d)Electronic mail: w.j.miloch@fys.uio.no

${ }^{\text {e)} E l e c t r o n i c ~ m a i l: ~ l a s s e . c l a u s e n @ f y s . u i o . n o ~}$

${ }^{\mathrm{f})}$ Electronic mail: saito@stp.isas.jaxa.jp

${ }^{\mathrm{g})}$ Electronic mail: yaqi.jin@fys.uio.no

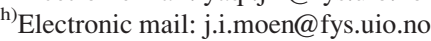

cannot be the source of the perturbations of the order of the ion gyroradius and other mechanisms are needed to explain the presence of such small-scale structures (e.g., Ref. 16).

One of the possible mechanisms to produce small-scale structures is the inhomogeneous energy-density-driven instability (IEDD instability). ${ }^{17}$ The theory of this instability was initially proposed by Refs. 17 and 18, where the authors considered an inhomogeneous $E \times B$ velocity profile in a singlelayer model, which generates electrostatic waves around the ion gyrofrequency: The inhomogeneous electric field is the source of free energy allowing for the instability development. Later, these results were expanded using other sources of free energy such as field-aligned velocity of particles ${ }^{19}$ and plasma density. ${ }^{20,21}$ Fluctuations of the electric field and of the plasma density were successfully identified in laboratory experiments with plasma (e.g., Refs. 22-25). It was proposed that the IEDD instability could be associated with auroral processes in the ionosphere (e.g., Refs. 26 and 27, and references herein). Numerical stability analysis using FAST or FREJA satellite data was carried out in recent papers, ${ }^{20,21,28-30}$ and it was confirmed that the IEDD instability could play an important role in the creation of electrostatic broadband turbulence (or noise) in the auroral zone and explain the experimental observations.

The physical mechanism of the IEDD instability is the following: inhomogeneities of the plasma background (in the presence of transverse velocity shear) may create regions with negative energy density, and a nonlocal wavepacket can couple the region of positive and negative energy, which can cause waves to grow (e.g., Ref. 31). It should be mentioned that the effect associated with the occurrence of negative energy density has been considered previously for example by Refs. 32 and 33, which studied wave interactions in the local approximation, and a discussion regarding the relationship 
between the excitation of waves with a negative energy and the anomalous Doppler effect can be found in Ref. 34. The IEDD instability can excite several types of waves, such as EIC and IA waves. ${ }^{19,20,28,30}$ An essential property of the IEDD instability is the broadband spectrum, so the application of the IEDD instability mechanism is of particular interest for ionospheric problems related to observations of broadband electrostatic waves (e.g., Refs. 16 and 27). The IA and EIC modes excited by the IEDD instability can lead to irregular small-scale plasma structures. One possible way for the IEDD mechanism to be developed is as a secondary process on a primary instability such as KHI; in other words, large-scale irregularities may provide a background on which the IEDD instability can evolve. ${ }^{16,31}$ Note that as the KHI is an hydrodynamic instability and is stabilized in the kinetic regime due to finite-Larmor-radius effects, and that the IEDD instability is not just a continuation of the classical KH oscillations toward the higher-frequencies and shorter-wavelengths. ${ }^{16,31}$

The IEDD instability is believed to take a principal part in the excitation of broadband electrostatic turbulence in the high-latitude ionosphere, ${ }^{20,21,35}$ and for ion energization and upflow from the ionosphere into the magnetosphere both in the cusp and auroral region. ${ }^{16,36}$ Indeed, ion upflows such as the ones occurring in the cleft ion fountain ${ }^{37}$ appear to be driven by processes operating at low altitudes where the intensity of field-aligned currents might not be large enough to excite current-driven electrostatic ion-cyclotron instabilities (CDEIC), ${ }^{16}$ and ion heating could occur due to the IEDD instability. ${ }^{19}$ It is interesting to note that correlations between ion upflows and reconnection events such as poleward moving auroral forms have been observed (e.g., Refs. 38 and 39). The cusp is a very complex area of precipitating, mirrored, and upwelling ions and electrons with the presence of magnetic and electric field perturbations and turbulence, which attest the importance of the cusp region in the transport of energy in magnetosphere-ionosphere coupling. ${ }^{40}$ The ionospheric plasma is particularly structured within the cusp region: using 18 months of ELF (extremely low frequency) electric field data from the DE-2 satellite. It was shown that the most intense low frequency $(4-8 \mathrm{~Hz})$ structures at high latitudes are those observed in the dayside cusp region. ${ }^{41}$ It was suggested that these electric field variations were either static structures or Alfven waves. Generally, the irregular wave structures observed in the cusp can be discussed either in the context of plasma instabilities triggered by flow of particles or by other inhomogenenous background, which create the flow of particles. ${ }^{42}$ Wave-particle interaction processes can generate the energy cascade from low-frequency waves to high-frequency waves via heating and acceleration of the plasma particles in the cusp. The strong intensity of the waves is associated with nonlinear processes initiated by wave-wave and/or wave-particle interactions. ${ }^{42}$

Lockwood et al. ${ }^{37}$ noticed persistent outflows of lowenergy $\mathrm{O}+$ ions and it was suggested that they had their origin in the dayside cleft/cusp region and manifested themselves as spatially dispersed field-aligned flows at higher altitude. The dayside cusp and the nightside auroral-polar cap boundary regions corresponding, respectively, to the low-altitude projections of dayside and nightside reconnection activity exhibit intense Alfven-wave turbulence, ion transverse heating and upward field-aligned ion flows. ${ }^{43}$ Observations have shown strong localized perpendicular energization of these ions, which can be attributed to wave-particle interactions. ${ }^{44}$ The energization has been associated with enhanced broadband extra low frequency wave power in the frequency range between $1 \mathrm{~Hz}$ and $1 \mathrm{kHz}$ (e.g., Refs. 36, 42, and 45). However, the free energy source for the wave growth and the identification of the wave modes responsible for ion heating are still open issues.

It was suggested that the IEDD instability could influence cusp and cleft dynamics (e.g., Ref. 16) but experimental and numerical works devoted to this question are still sparse. Thus, in order to investigate the role of the IEDD instability in the cusp ionosphere, we performed numerical stability analysis of the IEDD instability using data from the Investigation of Cusp Irregularities 3 (ICI-3) rocket as input parameters. The ICI-3 sounding rocket was launched from Ny-Ålesund $\left(78.9^{\circ} \mathrm{N}, 11.9^{\circ} \mathrm{E}\right.$, geographic coordinates) at 07:21:31 UT on December 3, 2011. ${ }^{46}$ The payload reached an apogee of $354.5 \mathrm{~km}$ after 312.9 seconds of flight, and its instruments consisted of AC and DC Electric field probes, a low-energy electron spectrometer (LEP) measuring energies in the range [0.01-7.9] keV, ${ }^{47}$ and magnetometers, as well as Langmuir probes. A scheme of the payload configuration showing the location of the different instruments as well as a description of the ICI-3 data can be found in Ref. 46.

The ICI- 3 rocket was launched into the cusp and crossed a reversed flow event (RFE), i.e., a $\sim 100-200 \mathrm{~km}$ wide quasistatic east-west elongated channel with flow direction in the opposite direction with respect to the background flow. ${ }^{4,48,49}$ The description of the RFE was initially introduced in Refs. 48 and 49. It was shown that the RFE phenomenon is a regular feature of the active cusp, which is likely connected to magnetic reconnection ${ }^{48}$ that could provide strong source of free energy, and therefore, an RFE is prolific for generation and evolution of various plasma instabilities (e.g., Refs. 4 and 50). Indeed, an RFE has irregular structures in wide range of scales on the entire region of RFE, not only on the edges of flow channels. ${ }^{46}$ These irregular structures have different characteristic lengths including meter and decameter scales, which are revealed by observing high-frequency spectral power during the event. Possible mechanisms explaining the existence of wide power spectra in relation to the RFE, which was observed by the rocket experiment, are proposed in our study. Suggestions that RFEs are accompanied by instability hierarchy are in accordance with this observation.

In the previous work, ${ }^{46}$ one particular region exhibiting small-scale electric field inhomogeneities was investigated and it was shown that the IEDD instability could be active and excite EIC waves; however, that paper mainly concerned the obtained rocket data and technical aspects of the rocket flight. In this study, we will investigate in detail the importance of the IEDD mechanism in different regions of the cusp intersected by the ICI-3 rocket and the influence of various sources of free energy such as shears in the particle drift velocity and inhomogeneous electric field.

In order to study the correlation between generation of the IEDD instability and irregular structures in the electric field, we use the Local Intermittency Measure (LIM), ${ }^{51}$ as 
the degree of irregularity dependences on scales. The LIM method was introduced as a new measure that can to estimate intermittency locally within a data sample. The LIM method uses the wavelet transform analysis, which consists of a mathematical technique capable of unfolding fluctuating quantities into scales (or frequencies) and time (or space). ${ }^{51,52}$

The structure of the article is organized as follows: The theoretical aspects of the IEDD instability theory in the presence of inhomogeneous field-aligned currents are considered in Sec. II. The computational methods used in this study and the analysis of the results are described in Sec. III. Section IV provides the discussion and conclusions.

\section{THEORETICAL ASPECTS}

In this section, we describe the physical mechanisms for the generation of the IEDD instability. The IEDD instability is a nonlocal instability in warm plasma driven by an inhomogeneous energy-density profile. It is nonlocal in the sense that it critically depends on spatial plasma inhomogeneities. ${ }^{18}$ In other words, the plasma behavior in one point also depends on the plasma behavior in remote points. This means that the IEDD instability cannot be simply analyzed in terms of algebraic dispersion relations $\omega(k)$ (frequency-wave vector) as it is the case for homogeneous plasma and, for such inhomogeneous plasmas, new approaches are required. For the case of the IEDD instability, the theory is based on the nonlocal approximation, and was initially developed in Refs. 16-18, and 31. Below, we recall the essential features of the IEDD instability theory following Ref. 17.

Let us consider perturbations of inhomogeneous magnetoactive plasma with the following configuration: An homogeneous magnetic field $\mathbf{B}$ oriented along the $z$ axis (corresponding to the Earth's magnetic field in the highlatitude ionosphere), a nonuniform electric field $\mathbf{E}(x)$ along the $x$ axis with plasma inhomogeneities in the $x$ direction as well. The magnetic and electric field create an $E \times B$ drift along the $y$ axis and the field-aligned velocity is directed along the magnetic field.

The dispersion relation for EIC waves takes the following form in the region, where the electric field is absent and where $k_{z} \rightarrow 0$ ( $k_{z}$ is the wave vector along the $z$ direction $):^{18}$

$$
D(\omega, k)=1-\Gamma_{0}-\sum_{n>0} \frac{2 \omega^{2} \Gamma_{n}(b)}{\omega^{2}-n^{2} \Omega_{i}^{2}}
$$

where $b=\left(k_{y} \rho_{i}\right)^{2} / 2$ is the normalized wave number in the $y$ direction, $\Omega_{i}=e \mathbf{B} / m_{i}$ is the ion gyrofrequency, $v_{t i}$ is the ion thermal velocity, $\rho_{i}$ is the ion gyroradius, $\Gamma_{n}(b)=\exp (-b) I_{n}$, and $I_{n}$ are the modified Bessel functions.

The energy density $U$ in the electrostatic wave is proportional to the $\partial(\omega D) / \partial \omega \approx \omega(\partial D / \partial \omega)$. Consequently, the energy density is defined by the relation

$$
U \sim \omega\left[\sum_{n>0} \frac{\omega n^{2} \Omega_{i}^{2} \Gamma_{n}(b)}{\left(\omega^{2}-n^{2} \Omega_{i}^{2}\right)^{2}}\right]=\omega^{2} \sigma(\omega),
$$

where $\sigma(\omega)>0$.
From Eq. (2), it is clear that the energy density is positive. If one adds an electric field $\mathbf{E}(x)$, an additional drift $V_{E}$ will occur along the y axis. In this case, the dynamical frequency in the formula (2) will be shifted by the Doppler effect: $\omega \rightarrow \omega_{1}=\omega-k_{y} V_{E}$. As a result, at sufficiently large values of $k_{y} V_{E}$, the energy density in the wave can become negative, and hence, we have

$$
U^{\prime} \sim \omega_{1} \omega \sigma\left(\omega_{1}\right)
$$

If the electric field is homogeneous, it is sufficient to transform into a frame of reference moving with a velocity $V_{E}$ along the $y$ axis in order to change the sign of the energy density in the wave, and retrieve the positive energy character. However, this becomes impossible when $V_{E}$ is inhomogeneous: For inhomogeneous $V_{E}$, the distribution of the energy density is inhomogeneous and the energy can be transferred from the region with a negative energy density to the region where the energy density is positive so that this energy flow can create the IEDD instability.

A rigorous study of a nonlocal instability in such a plasma configuration requires solving the Vlasov equations which are integro-differentials. Nevertheless, for some cases (smallness of dispersion and smallness of the Larmor radius compared to characteristic size of plasma inhomogeneity), the equations reduce to an ordinary differential equation ${ }^{31}$

$$
\frac{d^{2} \psi}{d \xi^{2}}+\kappa^{2}(\xi) \psi=0
$$

where the unknown function $\psi$ is the perturbation of the electric field potential, and where $\xi=x / \rho_{i}$ is the coordinate $x$ normalized to the ion gyroradius $\rho_{i}$. It is assumed that all inhomogeneities are along the $x$ axis, and

$$
\kappa^{2}(\xi)=-2 \frac{1+\sum_{n} \Gamma(b) F_{n i}(\xi)+\tau\left(1+F_{0 e}\right)}{\sum_{n} \Gamma^{\prime}(b) F_{n i}(\xi)} .
$$

Here, $F_{n \alpha}(\xi)=A_{n \alpha}(\xi)-B_{n \alpha}(\xi)$ and

$$
\begin{gathered}
A_{n \alpha}(\xi)=\frac{\left(\omega_{1 \alpha}+\omega_{2 \alpha}-\omega *_{\alpha}\right)}{\sqrt{2}\left|k_{z}\right| v_{t \alpha}} Z\left[\frac{\left(\omega_{1 \alpha}+\omega_{2 \alpha}-n \Omega_{\alpha}\right)}{\sqrt{2}\left|k_{z}\right| v_{t \alpha}}\right], \\
B_{n \alpha}(\xi)=\frac{\omega_{3 \alpha}}{2\left|k_{z}\right| v_{t \alpha}} Z^{\prime}\left[\frac{\left(\omega_{1 \alpha}+\omega_{2 \alpha}-n \Omega_{\alpha}\right)}{\sqrt{2}\left|k_{z}\right| v_{t \alpha}}\right] .
\end{gathered}
$$

Here, $\tau=T_{i} / T_{e}$ is the ratio of ion temperature to electron temperature, $v_{t \alpha}$ is the thermal velocity of particles of species $\alpha, \Omega_{\alpha}$ is the gyrofrequency of the particles of species $\alpha, \omega_{1 \alpha}=\omega-k_{y} V_{E}-k_{z} V_{d \alpha}$ is the Doppler shifted frequency, $V_{E}(\xi)$ is the velocity of the $E \times B$ drift, $\epsilon_{n}=\left[\rho_{\alpha} n_{0}(\xi)\right] /$ $\left(\frac{d n_{0}(\xi)}{d \xi}\right), \omega_{2 \alpha}=k_{y} V_{E}^{\prime \prime} \rho_{\alpha}^{2} / 2$ (where the symbol " signifies the second derivative), $\omega_{3 \alpha}=k_{y} V_{d \alpha}^{\prime} \rho_{\alpha}$ is the term which is responsible for the shear in field-aligned velocity, $V_{d \alpha}$ is the field-aligned velocity of species $\alpha, \Gamma_{n}^{\prime}=d \Gamma_{n} / d b$, and $Z$ is the plasma dispersion function. ${ }^{15}$ In the present study, we retain harmonics up to the second order for ions and 
harmonic $n=0$ for electrons, and $\Gamma(b)=1$ for $b \ll 1$. Detailed information regarding the choice of harmonics can be found in Ref. 31, and this approximation was validated by performing several numerical solutions using different numbers of harmonics and obtaining similar results.

To obtain the unstable frequencies, one has to find $\omega=\omega_{r}+i \gamma$ for which solution of the eigenvalue Eq. (4) exists and for which the growth rate $\gamma$ is positive. The solution must fade at infinity, i.e., $\psi( \pm \infty)=0$. The solution of the expression (4) $\psi$ depends on $\omega$ implicitly since $\omega$ occurs only in term $\kappa^{2}(\xi)$ in Eq. (4).

\section{NUMERICAL SOLUTIONS AND ANALYSIS OF THE RESULTS}

In order to solve the eigenvalue problem from Eq. (4), the shooting method is used. This method allows solving the boundary value problem by reducing it to the initial value problem. In numerical applications, it reduces differential equations to a system of algebraic equations, and can be done by many ways. The way used in this work is described below.

The interval considered for stability analysis is divided into layers, where $\kappa^{2}(\xi)$ is assumed to be constant. Thus, $\psi$ is the sum of two exponents in each layer, i.e., $\psi_{j}(\xi)$ $=B_{j} \exp \left(i \kappa_{j} \xi\right)+\widetilde{B}_{j} \exp \left(-i \kappa_{j} \xi\right)$, where $j$ is the number of layers, and $B_{j}$ and $\widetilde{B}_{j}$ are constant. For the first and the last layers, $\psi$ contains only one exponential and the sign of $\kappa_{j}$ corresponds to the boundary condition, i.e., the single term $\exp \left(i \kappa_{j} \xi\right)$ should become zero at infinity. It is also necessary that the solution obtained is continuously differentiable on the whole $x$ axis. This matching condition is satisfied by linear algebraic expressions containing coefficients before the exponentials, as shown in more detail in Ref. 21.

Hence, one has to solve the linear system with the unknown variables $B_{j}$ and $\widetilde{B}_{j}$, which can be represented in the matrix form

$$
M \cdot h=0,
$$

where $h$ is the vector composed of the coefficients $B_{j}$ and $\widetilde{B}_{j}$, the matrix $M$ is defined by the matching and boundary conditions. The exact expression of the matrix $M$, the boundary and matching conditions, and the computational methods are described in detail in our previous work. ${ }^{21}$

In the present study, we apply this method on the ICI-3 rocket data, which intersected a RFE. The RFE was observed using the EISCAT Svalbard Radar (ESR) 32m which was scanning in azimuth at a fixed elevation angle of $30^{\circ} .53$ The first evidence of the RFE was seen in the ESR scan of ion velocities starting at 07:24:11 UT, and the RFE was detectable in the two following scans, i.e., starting at 07:27:23 UT and 07:30:35 UT, respectively. Here, for completeness, we show in Fig. 1 the scan starting at 07:27:23 UT with the rocket trajectory superimposed as a magenta line. The magenta dots correspond to time steps of $100 \mathrm{~s}$ starting from launch time. The RFE is clearly visible as a red (away from the radar) velocity channel in a blue (towards the radar) background flow. The black segment on the trajectory corresponds to the time interval during which the radar completed

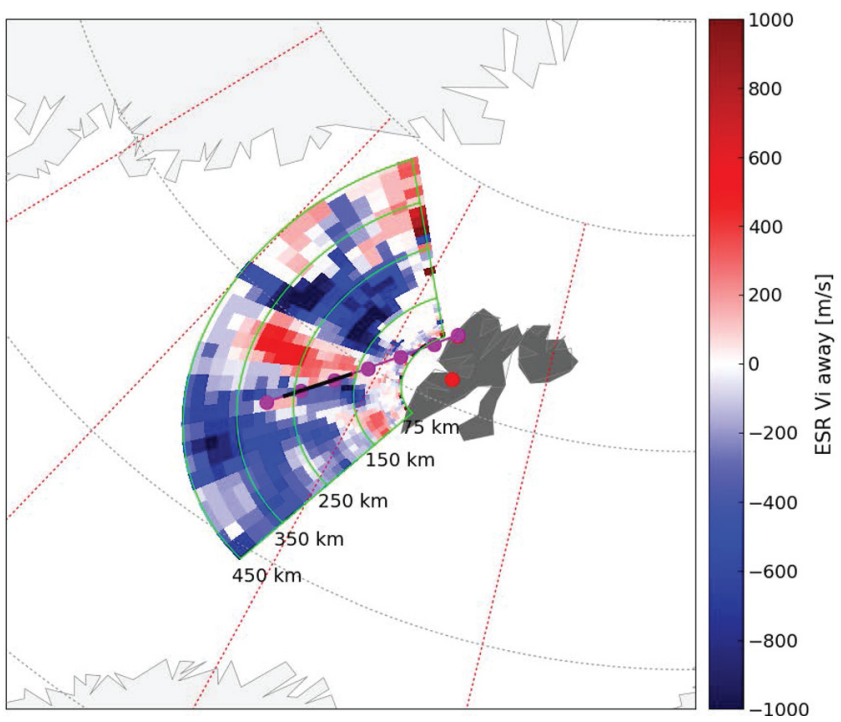

FIG. 1. EISCAT Svalbard radar fan plot of the ion velocities. The trajectory of the rocket is shown as a magenta line and the magenta dots correspond to time steps of $100 \mathrm{~s}$ starting from rocket launch time. The black portion of the trajectory corresponds to the time interval of the scan shown (07:27:23 UT to $07: 30: 35 \mathrm{UT}$ ). The altitudes of the radar beam are also shown.

the whole scan $(07: 27: 23$ UT to 07:30:35 UT). In addition, the altitudes of the radar beam are shown.

The measurements made by the ICI-3 sounding rocket with respect to time of flight between $t=150 \mathrm{~s}$ and $t=500 \mathrm{~s}$ are shown in Fig. 2. The corresponding altitudes are $h=236.8 \mathrm{~km}$ and $h=202.8 \mathrm{~km}$. The ICI-3 rocket intersected the RFE between about $t \sim 250 \mathrm{~s}$ and $t \sim 380 \mathrm{~s}_{\text {of flight. }}{ }^{46}$ Figure 2(a) shows the absolute electron density, which exhibits considerable fluctuations throughout the flight. The electron density was obtained using the multi-needle Langmuir probe system (m-NLP), ${ }^{54,55}$ and is shown here for completeness. In Fig. 2(b), the components of the electric field perpendicular to the magnetic field as well as its magnitude (black) are shown. The components are in the reference frame of the rocket, where it was assumed that the rocket was aligned with the magnetic field during the entire region investigated. The electric field observed during the ICI-3 flight was highly

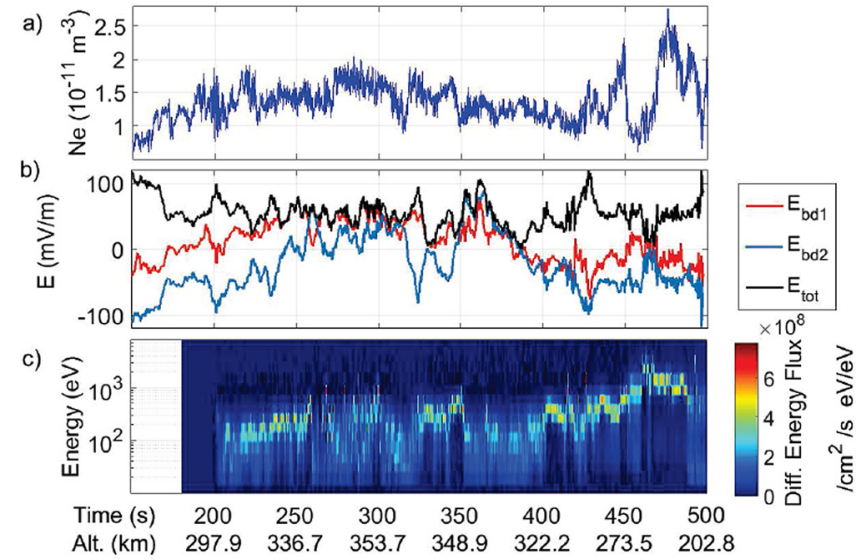

FIG. 2. ICI-3 sounding rocket measurements with respect to time of flight and altitude: (a) Electron density. (b) Components (red and blue) and magnitude (black) of the electric field. (c) Electron particle precipitation flux at $0^{\circ}-30^{\circ}$ pitch angle. 
structured, with and several direction changes and gradients. The distribution of the electron particle precipitation flux at $0^{\circ}-30^{\circ}$ pitch angle, i.e., along the magnetic field lines, measured on-board the ICI-3 rocket is presented in Fig. 2(c). The energies and differential energy fluxes are shown in the vertical and color axis, respectively. The presence of electron precipitation can be observed during most of the time interval presented in Fig. 2, with an increase in energies at the end, where they reach $7 \mathrm{keV}$.

As noted above, a numerical analysis of the IEDD instability is conducted using ICI-3 sounding rocket data as input parameters. We consider one component of the electric field in the rocket reference frame (the blue component in Fig. 2) and we smoothed fluctuations by averaging the electric field in each of the 30 layers used for the numerical analysis (a figure showing how this averaging is performed can be found in Ref. 21). In addition, we consider the field-aligned velocity of particles in our numerical stability analysis. We assume that the field-aligned velocity is formed from both the electrons and ions. To obtain this velocity, we use the rocket particle precipitation data in the following way. The energy flow of electrons is converted to the velocity, which is then normalized using the average velocity of electrons. For the ion velocity, we adopt $V_{d i}(\xi)=0.02 V_{d e}(\xi)$, which is approximately consistent with the ratio of ion mobility to electron mobility in the Earth's ionosphere. ${ }^{30}$ Since it appeared that IEDD waves are destabilized more readily for $O+$ ions, that is, for a wider range of the input parameters, ${ }^{56}$ we present the results for this particular species.

Before we proceed with the results, it is worth noting some limitations associated with the calculations presented here. The first one is related to the different components of the electric fields. In the theory, the inhomogeneities are only along a fixed $x$ axis and the model is one dimensional. As the flow direction (and components) was changing a lot during the different intervals, and due to uncertainties in estimating precisely the flow direction, we investigate one component of the electric field in the rocket reference frame. The component selected is the one exhibiting more irregularities. As we are mainly interested in qualitatively detecting the presence of the IEDD instability, the selection of the reference frame is not crucial and, in general, we expect that the second component would increase the growth rate of the IEDD instability. Also, the smoothing of the data in different layers is not critical for the results, as the growth rates obtained are expected to be greater for the original (unsmoothed) data, because in general case various shears would enhance the instability. ${ }^{57}$ Thus, the results considering both components and unsmoothed would accentuate the results presented here.

Another limitation is that the electric field measured corresponds to a steady state. The IEDD instability growth reduces the magnitude of the electric field and enlarges its scale size, that is, it reduces the shear in the nonlinear stage and, hence, it affects the inhomogeneity and reduces the ExB flow. Paper ${ }^{58}$ showed that the reduction in the electric field magnitude is due to a second order polarization drift along the electric field. Then, the numerical results of PIC simulations ${ }^{59,60}$ demonstrated both reduction in magnitude and enlargement in the scale size of the electric field. If an electric pulse is applied to plasma, the IEDD instability would grow and reduce its magnitude down to the threshold at which point the emission will stop. But the ionosphere is a driven system where the electric field is constantly reinforced by the magnetospheric boundary condition (as in Nishikawa's simulations mentioned above). This results in a steady state balance between the waves and the electric field at saturation. The sounding rocket observes this steady state. Furthermore, it is not straightforward to answer the questions regarding the importance of the damping rate during RFE and here, we focus on the IEED instability growth.

Having in mind the assumptions given above, we perform IEDD instability analysis on the ICI-3 electric field data. We first consider two different time intervals, i.e., $\Delta t_{A}$ $=[338-358] \mathrm{s}$ and $\Delta t_{B}=[453-473] \mathrm{s}$. We chose these intervals because they exhibit different profiles of the electric field, as can be seen in Fig. 2: the electric field in $\Delta t_{A}$ exhibits large scale gradients, while more smaller-scale structures are present in interval $\Delta t_{B}$. Furthermore, the field-aligned velocity is larger in the latter interval, as can be inferred from the precipitation data shown in Fig. 2. Using these two intervals, we can investigate the role of electric field inhomogeneities perpendicular to the magnetic field as well as the effect of field aligned particle velocity inhomogeneities on the growth rate.

Surface plots with coordinates $\left(b, \omega_{r}\right)$ are used to present the results of the numerical analysis, with the normalized growth rate $\gamma$ shown in color. The results for the EIC branch for the time interval $\Delta t_{A}$ are presented in Fig. 3. Figure 3(a) shows the modeling results when only the inhomogeneous electric field is taken into account, and Fig. 3(b) shows the results when both inhomogeneous field-aligned plasma flow and inhomogeneous electric field are used as input parameters for the numerical analysis. The background plasma parameters used for the numerical stability analysis are the following: $\tau=0.5, u=0.1$, and $\rho_{i}=5 \mathrm{~m}\left(u=k_{z} / k_{y}\right.$ is the ratio of wave numbers along $z$ and $y$ directions).

As can be seen in Fig. 3, the unstable solutions are present for various values of the frequency $\omega_{r}$ and parameter $b$ (normalized wavenumber in the $y$ direction). In general, the unstable solutions are localized along parabolic curves and the growth rate increases with the increase in $b$. Moreover, the unstable solutions obtained do not form isolated groups and can generate a broadband spectrum. By

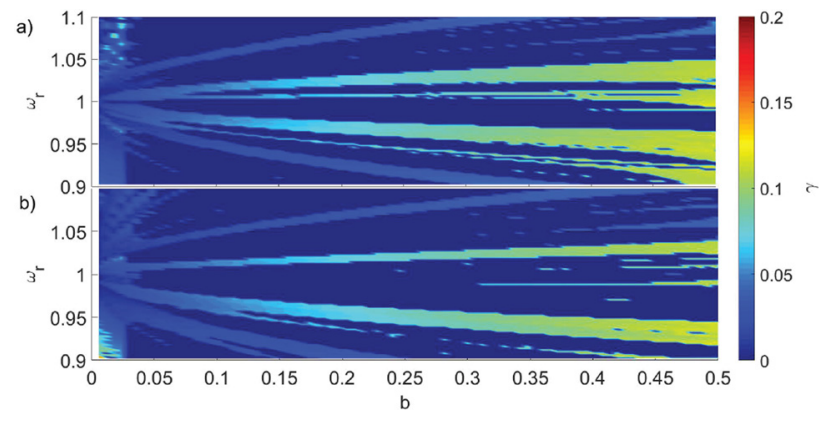

FIG. 3. Growth rate $\gamma$ of the IEED instability normalized by the oxygen ion gyrofrequency depending on the parameter $b$ and the real part of the wave frequency $\omega_{r}$ in the time interval $\Delta t=[338-358]$ (a) using the D.C. electric field data measured by the ICI-3 sounding rocket in the interval and (b) with addition field-aligned particle velocity. 


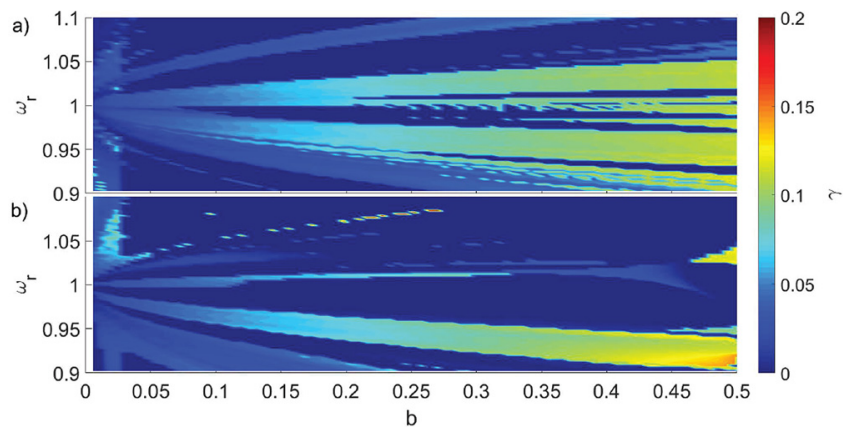

FIG. 4. Growth rate $\gamma$ of the IEED instability normalized by the oxygen ion gyrofrequency depending on the parameter $b$ and the real part of the wave frequency $\omega_{r}$ in the time interval $\Delta t=[453-473]$ (a) using the D.C. electric field data measured by the ICI-3 sounding rocket in the interval and (b) with addition field-aligned particle velocity.

comparing Figs. 3(a) and 3(b), one can see that the inclusion of the field-aligned particle velocity does not change much the results of the numerical analysis for interval $\Delta t_{A}$, except that it slightly decreases the unstable domains, i.e., the nonlocal EIC waves are excited for a smaller set of frequencies and wave numbers. Note that plasma density inhomogeneities were not included in the computations as their influence on the IEDD instability development has been shown to be negligible. $^{21}$

The results for $\Delta t_{B}$ are shown in Fig. 4, which corresponds to the time interval during which the rocket was equatorward of the RFE (see Fig. 1 and Ref. 46). Figure 4(a) presents the growth rates obtained using only the electric field data as inputs for the analysis and in Fig. 4(b), the velocities obtained from the particle precipitation are included. Again, the unstable solutions are localized along parabolic curves and increase with respect to $b$. Furthermore, it can be inferred from Fig. 4(b) that including shears in field-aligned velocity strongly decreases the growth rate of the IEDD instability. This decrease in unstable domains for the creation of EIC waves by the IEDD instability seems to be a common feature for the ICI-3, as we checked several intervals exhibiting similar results (not shown).

When comparing Fig. 3 ( $\Delta t_{A}$ : large-scale gradients in $\mathrm{E}$ ) and Fig. 4 ( $\Delta t_{B}$ : several smaller-scale gradients of $\left.\mathrm{E}\right)$, we can see that the unstable modes fill broader areas along curves in the latter case. The growth rate of the IEDD instability is thus facilitated in the presence of several gradients in the electric field at different scales. Furthermore, the presence of stronger field-aligned velocity reduces the growth rate more dramatically (case $\Delta t_{B}$ ).

Now that two intervals with different characteristics in the electric field and field aligned velocity were presented in detail, we investigate the growth rates for the entire flight. For visualization purposes, Figs. 5(a) and (b) show the component of the electric field measured by the sounding rocket. The figure is divided due to a gap in the electric field data at 316-320 s of rocket flight. Panel a) roughly corresponds to the upleg and panel b to the downleg (see Fig. 2). The electric field data were divided into intervals of $20 \mathrm{~s}$ with $10 \mathrm{~s}$ overlap. For each interval, the growth rate of the IEDD instability and the mean values of the growth rate over $\omega_{r}$ were calculated. The mean growth rates are plotted in color with respect to time (horizontal axis) and with $b$ (vertical axis) in Figs. 5(c) and 5(d). Note that the values shown at each time correspond to the growth mean rate obtained for the $20 \mathrm{~s}$ intervals following that time. Several regions of the flight exhibit larger growth rates, with the largest for the intervals around $t=[270,290] \mathrm{s}$, and around $t=[450-470] \mathrm{s}$. In these two intervals, the electric field is quite inhomogeneous, with the presence of many structures. Comparing Figs. 5(a) and 5(b) with Figs. 5(c) and 5(d), one can infer that the presence of several irregular structures facilitates the creation of EIC waves in comparison with regions with large-scale gradients (such as the one at about $260 \mathrm{~s}$ ). This is in agreement with the conclusions of the paper. ${ }^{57}$

To investigate the relation between inhomogeneities and IEDD instability growth rates in more detail, we performed a LIM analysis using wavelet transforms. ${ }^{51,61}$ The LIM is defined as

$$
\operatorname{LIM}(s, t) \equiv \frac{\left|W_{s}(t)\right|^{2}}{\left\langle\left|W_{s}(t)\right|^{2}\right\rangle_{t}},
$$

where $\langle\ldots\rangle_{t}$ corresponds to the averaging over time, and $W_{s}(t)$ are the wavelet coefficients for the electric field at scales $s$ and time $t .^{51,62,63}$ Here, the wavelet coefficient calculations were adapted from a software provided by Ref. 52
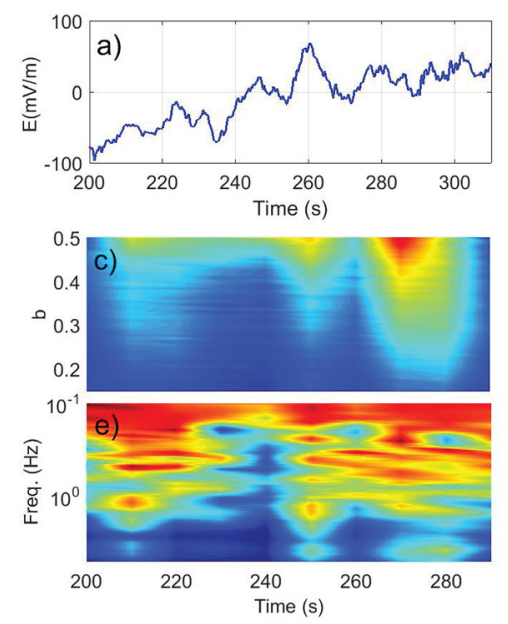
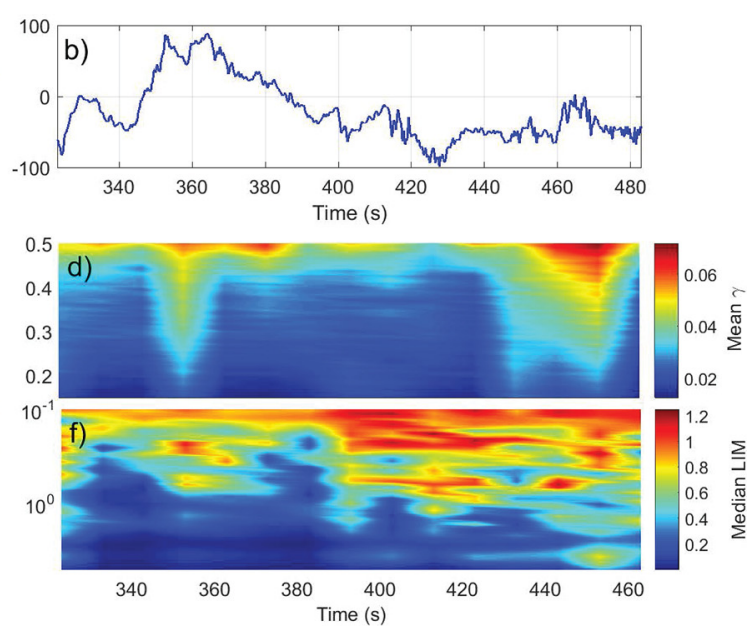

FIG. 5. (a) and (b) Electric field data measured by ICI-3. (c) and (d) Growth rate of the IEED instability normalized by the oxygen ion gyrofrequency depending on the parameter $b$ averaged over the real part of the wave frequency $\omega_{r}$ for intervals of $20 \mathrm{~s}$ with $10 \mathrm{~s}$ overlap. (e) and (f) Median of the LIM for the same intervals as in panel (c) and (d). 
using Morlet wavelets, and further details on wavelet analysis can be found in Refs. 51, 52, and 64. The LIM quantity measures the relative power among wavelets at a particular scale, or in other words, the local activity of the signal at each scale $s:{ }^{63}$ As $\left\langle\left|W_{s}(t)\right|^{2}\right\rangle_{t}$ is equivalent to the Fourier spectrum, the condition $L I M=1$ means that each part of the signal has the same energy spectrum as the Fourier spectrum and that the signal does not exhibit intermittency, which is characterized by the degree of non-uniformity of the wavelet power. ${ }^{61,62}$ On the other hand, values of the LIM greater than one indicate intermittency. ${ }^{63}$

For each of the $20 \mathrm{~s}$ interval, the LIM was calculated for the electric field $E_{L I M}(t)=E(t)-E\left(t_{0}\right)$, where $t_{0}$ is the time in the beginning of each intervals (this was done to match the normalization used for the numerical analysis). For comparison with the growth rates of the IEDD instability and to obtain a rough estimate of the LIM in each interval, the median of the LIM at each scale was calculated for each interval. The median LIM at each scale is plotted in Figs. 5(e) and 5(f) with respect to time and frequency, which ranges from $0.1 \mathrm{~Hz}$ to $10 \mathrm{~Hz}$ due to restrictions associated with the cone of influence at lower frequency ${ }^{52}$ and the electric field resolution. Again, the values shown at each time correspond to the median LIM obtained for the $20 \mathrm{~s}$ intervals following that time. Several regions with enhancements in the LIM over many frequency scales can be observed, in particular at about $210 \mathrm{~s}, 250 \mathrm{~s}, 270 \mathrm{~s}, 350 \mathrm{~s}$, and at the end of the flight, implying the presence of the enhanced "local activity" of the electric field in those intervals for several frequencies (or scales). By comparing Figs. 5(c) and 5(d) with Figs. 5(e) and 5(f), one can observe a qualitative agreement between those regions with enhanced growth rate values and those with enhanced LIM. In particular, all the intervals with a large growth rate relate to enhancements in the LIM, and thus in electric field inhomogeneities. In other words, the IEDD instability exists in turbulent intermittent (or nonGaussian) process in the cusp. It should however be noted that the presence of LIM does not necessary always imply that IEDD instability is active, as visible for example at about 410 s. Perhaps, in this case, there are other plasma instabilities that are responsible for irregular processes.

\section{DISCUSSION}

The IEDD instability was proposed to be a good candidate for explaining micro-scales effects in high-latitude ionosphere. ${ }^{16}$ This plasma instability is developed in the presence of inhomogeneous energy density, and inhomogeneous electric field, plasma density gradients, and shears in the particle parallel drift velocity could be possible sources of free energy necessary for the IEDD instability to develop (e.g., Refs. 17, 19-21). It was shown that inhomogeneous structures of electric field and field-aligned current produce broadband spectra for several wave modes such as EIC ${ }^{21}$ and $\mathrm{IA}^{30}$ waves. The role of the IEDD instability could also be broader than the production of broadband waves in the auroral zone. The IEDD instability may be engaged into the particle energization and ion outflow that take part in the energy transfer between the magnetosphere and ionosphere. ${ }^{16}$ Thus, it is of particular interest to investigate the occurrence and driving mechanisms of the IEDD instability not only in the auroral ionosphere, but also in the cusp. The IEDD instability has been well discussed in the literature, but little tested against potentially definitive data. In this study, we performed numerical stability analysis using in situ data measured by the ICI-3 sounding rocket, which intersected an RFE with a strongly fluctuating plasma environment. This makes the ICI-3 data well suited for such a test.

There are many processes in the cusp ionosphere that require a micro-scale kinetic approach, such as the investigation of the IEDD instability, since the cusp is the region where the magnetic barrier is small enough to allow the magnetosheath plasma to participate in the global energy/ momentum transfer. ${ }^{16,65}$ One of the examples of complex processes in the cusp where both large- and micro- scales phenomena play an important role is the RFE, where a lot of density and electric field inhomogeneities can be encountered (e.g., Refs. 46 and 50). The suggestion that RFEs are accompanied by instability hierarchy is in accordance with its highly inhomogeneous structures.

The IEDD instability is a microscale instability that can be generated as secondary instability, since it requires macro- or meso-scales inhomogeneities. For example, in the case of the auroral zone, the IEDD instability was generated by electric field inhomogeneities created by coherent alfvenic structures and shear in magnetospheric convection. There are some possible candidates for the source of the inhomogeneous energy density profile including the KHI, the D'Angelo instability, ${ }^{16}$ and the GDI. All of these instabilities provide inhomogeneous mesoscale background and it is rather difficult to recognize the precise contribution of each of them. Nevertheless, all of them might be present in the RFE evolution. The role and interplay of the GDI and of the KHI were for example discussed in Refs. 4 and 8. The D'Angelo instability requires shear in the flow along the ambient magnetic field that can occur in the RFE. However, it is likely that the most appropriate mesoscale instability for development of the IEDD instability is the KHI. Perhaps, velocity-sheared fluid-like processes, in the form of the KHI, develop into strongly steepened structures due to nonlinear convective effects: ${ }^{16}$ These steepened structures can drive smaller-scale secondary instabilities such as the IEDD instability. Moreover, the IEDD instability is closely related to the KHI because the equation describing the IEDD mechanism transforms into the KHI equation in the limit of long wavelengths and low frequency. ${ }^{16}$ However, the IEDD instability is not kinetic continuation of the KHI, since the KHI suffers from finite Larmor radius stabilization. ${ }^{16}$ The KHI is maintained by the gradient of the velocity shear $d^{2} V_{E} / d x^{2}$, whereas this is unnecessary for development of the IEDD instability because the IEDD instability is provided by inhomogeneous energy density distribution.

In this study, we tested the potential role of the IEDD instability using ICI-3 data for different energy sources. We have shown that the IEDD instability is active both in the proximity of the RFE and in its center. The possibility of IEDD instability generation is different in each interval; however, the instability domain is noticeable in each case. 
Thus, it supports the idea that microscale irregularities associated with RFEs are connected with the action of this instability. We investigated two cases with different configurations of both the perpendicular electric field and the field aligned velocity, and showed that the IEDD instability growth rate is facilitated for inhomogeneous electric field, and that it is strongly affected for enhanced field-aligned velocities, i.e., reduced in the case considered [see Figs. (3) and (4)]. It is shown that IEDD instability takes part in the irregular processes during RFE. The obtained results demonstrate that the broadband wave generation by the IEDD mechanism is rather general and is characteristic of various ionospheric regions. Besides, we performed the calculations for many intervals along the rocket flight and, in general, the growth rate changes when the field-aligned current is added in our modeling (not shown). Consequently, the influence of the field-aligned velocity shear of ions depends on the profile of the field-aligned velocity and of the electric field. This result is consistent with the previous work, ${ }^{28}$ where quantitative analysis of ionospheric solitary structures in the auroral zone was provided, and where it was demonstrated that the dominating branch of the electrostatic turbulence is determined by the interplay of various driving sources inside a particular solitary structure. The sources do not generally act in unison, so that their common effect may be inhibiting for excitation of electrostatic waves of a certain type.

To further investigate the relation between inhomogeneities in the electric field and the growth rate of the IEDD instability, we also performed a LIM analysis. The LIM has already shown its usability in previous turbulence studies such as the study of solar wind turbulence (e.g., Ref. 61), an intermittency analysis of the electric field measured by a sounding rocket in the auroral zone, ${ }^{62}$ and the study of the Auroral Electrojet index. ${ }^{63}$ Here, we apply it to cusp ionospheric turbulence, and we show that there is an agreement between the IEDD instability generation and irregular structures of the cusp. It should be noted that the best correlations occur in the cases where irregularities can be observed over several scales (versus the presence of only large-scale gradients), although the calculations of the growth rate used only low-scale electric field (i.e., smoothed over the 30 layers). Also, one can see that large growth rate values of the IEDD instability are always accompanied by large values of LIM. It can be an additional argument for the presence of the IEDD instability, among other instabilities, to be active in creation of turbulence in the cusp. In the regions where the growth rate of the IEDD instability is low and LIM is rather high, other instabilities may be dominant, such as the $\mathrm{KH}$ instability. Such an agreement between the generation of the IEDD instability and RFE irregular structures implies that the IEDD instability may be connected with turbulent nonGaussian processes in the cusp. In general, the LIM estimates intermittency within a data sample, making it useful for such an inhomogeneous region such as the RFE, and can be used to study intermittent and non-Gaussian processes in ionospheric plasma. In particular, the fact that we can compare growth rates and the LIM encourages the use of LIM for further studies of ionospheric instabilities.
It should be noted that the IEDD instability produces waves at various frequencies. The presence of energy density inhomogeneities may be considered as a way of broadening spectra of classical modes in a homogeneous warm plasma. ${ }^{16}$ This leads to a broadband spectrum and simplifies wave excitation. In other words, the IEDD instability is generated in a wider range of plasma parameters than instabilities in homogeneous media, for instance, CDEIC (current-driven electrostatic ion cyclotron) instability. ${ }^{9}$ Thus, the results obtained provide good arguments to expect that the IEDD instability develops on wider interval of frequencies and can explain the cusp observations from sounding rockets. This generally supports the idea that microscale irregularities in cusp flow channels such as RFEs are connected with the action of micro-scale (kinetic) instabilities such as the IEDD instability, which can drive the plasma unstable on the background evolution of a primary instability such as the KHI.

\section{CONCLUSION}

We have shown that the IEDD instability is active in the cusp ionosphere, both on the sides and in the center of the RFE. This supports the idea that microscale irregularities in cusp flow events (such as RFEs) are connected with the action of this instability. A secondary generation of microscale inhomogeneities by the IEDD instability on the background evolution of a primary instability such as KHI might thus be well suited for describing RFE irregularities, where the electric field is quite inhomogeneous. We further showed that the IEDD instability growth rate is facilitated in the presence of enhanced inhomogeneities in the electric field, and that it is strongly affected by field-aligned velocities. This was supported by a LIM analysis, which was tested for the first time using cusp rocket data in this study and it was proved that the LIM method is applicable to the physics of the ionosphere. LIM estimates intermittency and it is useful for such an inhomogeneous region such as the RFE. LIM analysis has shown that the large growth rate of the IEDD instability corresponded to high values of LIM at different scales, indicating that the IEDD instability, among other instabilities, was active in creation of turbulence in the cusp. In the regions where the growth rate of the IEDD instability is low and LIM is rather high, perhaps, other plasma instabilities are dominant, for instance, KHI. According to LIM analysis, such an agreement between the generation of the IEDD instability and RFE irregular structures suggests that the IEDD instability may be connected with turbulent nonGaussian processes in the cusp ionosphere.

\section{ACKNOWLEDGMENTS}

This work has been supported by the Norwegian Center for International Cooperation in Education (SIU), Grant No. UTF-2016-short-term/10026. This project has also been supported by the Research Council of Norway Grant Nos. 208006 and 230996, and is a part of the 4DSpace Strategic Research Initiative at the University of Oslo. This study was supported by the Russian Science Foundation (Grant No. 17-77-20009). We acknowledge fruitful discussions of the results by the study team at the International Space Science 
Institute (ISSI). We acknowledge Andya Space Centre for providing payload services throughout the project period and launch operations of the ICI-3 rocket. The payload services and the launch operations were funded through the Norwegian Space Center. EISCAT is an international association supported by research organisations in China (CRIRP), Finland (SA), Japan (NIPR and STEL), Norway (NFR), Sweden (VR), and the United Kingdom (NERC). The m-NLP electron density and the electric field data from the ICI-3 rocket can be obtained on request from Lasse Clausen (lasse.clausen@fys.uio.no) and data from the electron particle experiment can be requested from Yoshifumi Saito (saito@stp.isas.jaxa.jp). The wavelet coefficients were calculated based on the wavelet software provided by C. Torrence and G. Compo, and are available at URL: http://atoc.colorado.edu/research/wavelets/.

${ }^{1}$ M. J. Keskinen and S. L. Ossakow, Radio Sci. 18, 1077, https://doi.org/ 10.1029/RS018i006p01077 (1983).

${ }^{2}$ R. T. Tsunoda, Rev. Geophys. 26, 719, https://doi.org/10.1029/ RG026i004p00719 (1988).

${ }^{3}$ G. Paschmann, S. Haaland, R. Treumann, O. Amm, J. Birn, J. Bonnell, J. Borovsky, J. Carbary, C. Carlson, C. Cattell, R. Ergun, A. Eriksson, Y. Galperin, T. Hallinan, G. Haerendel, B. Hultqvist, T. Karlsson, D. Knudsen, H. Koskinen, W. Lotko, G. Lu, K. Lynch, R. Lysak, G. Marklund, J. McFadden, A. Masson, C.-I. Meng, H. Opgenoorth, R. Pottelette, T. Pulkkinen, V. Sergeev, and Y. Song, Space Sci. Rev. 103, i (2002).

${ }^{4}$ J. Moen, K. Oksavik, L. Alfonsi, Y. Daabakk, V. Romano, and L. Spogli, J. Space Weather Space Clim. 3, A02 (2013).

${ }^{5}$ P. M. Kintner, B. M. Ledvina, and E. R. de Paula, Space Weather 5, s09003, https://10.1029/2006SW000260 (2007).

${ }^{6}$ P. Prikryl, P. T. Jayachandran, S. C. Mushini, and R. Chadwick, Ann. Geophys. 29, 377 (2011).

${ }^{7}$ Y. Jin, J. I. Moen, and W. J. Miloch, J. Space Weather Space Clim. 4, A23 (2014).

${ }^{8}$ K. Oksavik, J. Moen, M. Lester, T. A. Bekkeng, and J. K. Bekkeng, J. Geophys. Res.: Space Phys. 117, A11301, https://10.1029/ 2012JA017835 (2012).

${ }^{9}$ J. M. Kindel and C. F. Kennel, J. Geophys. Res. 76, 3055, https://doi.org/ 10.1029/JA076i013p03055 (1971).

${ }^{10}$ M. C. Kelley and C. W. Carlson, J. Geophys. Res. 82, 2343, https:// doi.org/10.1029/JA082i016p02343 (1977).

${ }^{11}$ M. J. Keskinen, H. G. Mitchell, J. A. Fedder, P. Satyanarayana, S. T. Zalesak, and J. D. Huba, J. Geophys. Res.: Space Phys. 93, 137, https:// doi.org/10.1029/JA093iA01p00137 (1988).

${ }^{12}$ J. Moen, I. K. Walker, L. Kersley, and S. E. Milan, J. Geophys. Res.: Space Phys. 107, 1044, https://doi.org/10.1029/2001JA000111 (2002).

${ }^{13}$ G. D. Earle, M. C. Kelley, and G. Ganguli, J. Geophys. Res.: Space Phys. 94, 15321, https://doi.org/10.1029/JA094iA11p15321 (1989).

${ }^{14} \mathrm{~S}$. Chandrasekhar, Hydrodynamic and Hydromagnetic Stability (Dover Publications, 1961), p. 652.

${ }^{15}$ A. B. Mikhailovskii, Theory of Plasma Instabilities, Vol. 2 (Springer US, 1974), p. 314.

${ }^{16}$ G. Ganguli, M. J. Keskinen, H. Romero, R. Heelis, T. Moore, and C. Pollock, J. Geophys. Res. 99, 8873, https://doi.org/10.1029/93JA03181 (1994).

${ }^{17}$ G. Ganguli, Y. C. Lee, and P. G. Palmadesso, Geophys. Res. Lett. 12, 643, https://doi.org/10.1029/GL012i010p00643 (1985).

${ }^{18}$ G. Ganguli, Y. C. Lee, and P. G. Palmadesso, Phys. Fluids 28, 761 (1985).

${ }^{19}$ V. Gavrishchaka, M. E. Koepke, and G. Ganguli, Phys. Plasmas 3, 3091 (1996).

${ }^{20}$ A. Chernyshov, A. Ilyasov, M. Mogilevsky, I. Golovchanskaya, and B. Kozelov, Plasma Phys. Rep. 41, 254 (2015).

${ }^{21}$ A. A. Ilyasov, A. A. Chernyshov, M. M. Mogilevsky, I. V. Golovchanskaya, and B. V. Kozelov, Phys. Plasmas 22, 032906 (2015).

${ }^{22}$ M. E. Koepke, W. E. Amatucci, J. J. I. Carroll, and T. E. Sheridan, Phys. Rev. Lett. 72, 3355 (1994)

${ }^{23}$ W. E. Amatucci, D. N. Walker, G. Ganguli, D. Duncan, J. A. Antoniades, J. H. Bowles, V. Gavrishchaka, and M. E. Koepke, J. Geophys. Res. 103, 11711, https://doi.org/10.1029/98JA00659 (1998).
${ }^{24}$ M. E. Koepke, W. E. Amatucci, J. J. Carroll III, V. Gavrishchaka, and G. Ganguli, Phys. Plasmas 2, 2523 (1995).

${ }^{25}$ Y. Liu, J. Lei, P. Yu, Z. Zhang, X. Zhang, and J. Cao, Geophys. Res. Lett. 44, 1634, https://doi.org/10.1002/2016GL072232 (2017).

${ }^{26}$ M. Hamrin, P. Norqvist, M. Andre, and A. Eriksson, J. Geophys. Res.: Space Phys. 107, SIA 21-1, https://10.1029/2001JA900144 (2002).

${ }^{27}$ P. M. Kintner, J. Franz, P. Schuck, and E. Klatt, J. Geophys. Res.: Space Phys. 105, 21237, https://doi.org/10.1029/1999JA000323 (2000).

${ }^{28}$ I. V. Golovchanskaya, B. V. Kozelov, A. A. Chernyshov, M. M. Mogilevsky, and A. A. Ilyasov, Phys. Plasmas 21, 082903 (2014).

${ }^{29}$ A. Chernyshov, A. Ilyasov, M. Mogilevsky, I. Golovchanskaya, and B. Kozelov, Cosmic Res. 54, 52 (2016).

${ }^{30}$ A. A. Ilyasov, A. A. Chernyshov, M. M. Mogilevsky, I. V. Golovchanskaya, and B. V. Kozelov, J. Geophys. Res.: Space Phys. 121, 2693, https://doi.org/10.1002/2015JA022117 (2016).

${ }^{31}$ G. Ganguli, Y. C. Lee, and P. G. Palmadesso, Phys. Fluids 31, 823 (1988).

${ }^{32}$ A. Bers and S. Gruber, Appl. Phys. Lett. 6, 27 (1965).

${ }^{33}$ B. B. Kadomtsev, A. B. Mikhailovskii, and A. V. Timofeev, JETP 20, 1517 (1965).

${ }^{34}$ M. V. Nezlin, Phys. Usp. 19, 946 (1976).

${ }^{35}$ A. Chernyshov, A. Ilyasov, M. Mogilevsky, I. Golovchanskaya, and B. Kozelov, Geomagn. Aeron. 57, 308, https://doi.org/10.1134/ S0016793217030045 (2017).

${ }^{36}$ M. André, P. Norqvist, L. Andersson, L. Eliasson, A. I. Eriksson, L. Blomberg, R. E. Erlandson, and J. Waldemark, J. Geophys. Res. 103, 4199, https://doi.org/10.1029/97JA00855 (1998).

${ }^{37}$ M. Lockwood, M. O. Chandler, J. L. Horwitz, J. H. Waite, T. E. Moore, and C. R. Chappell, J. Geophys. Res.: Space Phys. 90, 9736, https:// doi.org/10.1029/JA090iA10p09736 (1985).

${ }^{38}$ J. Moen, K. Oksavik, and H. C. Carlson, Geophys. Res. Lett. 31, L11808 https://doi.org/10.1029/2004GL020129 (2004).

${ }^{39}$ A. Skjaeveland, J. Moen, and H. C. Carlson, J. Geophys. Res.: Space Phys. 116, A10305, https://10.1029/2011JA016480 (2011).

${ }^{40}$ P. J. Cargill, B. Lavraud, C. J. Owen, B. Grison, M. W. Dunlop, N. Cornilleau-Wehrlin, C. P. Escoubet, G. Paschmann, T. D. Phan, L. Rezeau, Y. Bogdanova, and K. Nykyri, "Cluster at the magnetospheric cusps," in Outer Magnetospheric Boundaries: Cluster Results, edited by G. Paschmann, S. J. Schwartz, C. P. Escoubet, and S. Haaland (Springer Netherlands, Dordrecht, 2005), pp. 321-366.

${ }^{41}$ J. P. Heppner, M. C. Liebrecht, N. C. Maynard, and R. F. Pfaff, J. Geophys. Res.: Space Phys. 98, 1629, https://doi.org/10.1029/92JA01836 (1993).

${ }^{42}$ The Magnetospheric Cusps: Structure and Dynamics, edited by T. A. Fritz and S. F. Fung (Springer Netherlands, 2005), p. 414

${ }^{43}$ W. Lotko, J. Atmos. Sol.-Terr. Phys. 69, 191 (2007).

${ }^{44}$ T. E. Moore, R. Lundin, D. Alcayde, M. André, S. B. Ganguli, M. Temerin, and A. Yau, Space Sci. Rev. 88, 7 (1999).

${ }^{45}$ K. S. Jacobsen and J. I. Moen, Ann. Geophys. 28, 1249 (2010).

${ }^{46}$ A. Spicher, A. A. Ilyasov, W. J. Miloch, A. A. Chernyshov, L. B. N. Clausen, J. I. Moen, T. Abe, and Y. Saito, J. Geophys. Res.: Space Phys. 121, 10466, https://doi.org/10.1002/2016JA022999 (2016).

${ }^{47}$ Y. Saito, S. Yokota, K. Asamura, and A. Krieger, J. Geophys. Res.: Space Phys. 122, 1816, https://doi.org/10.1002/2016JA023157 (2017).

${ }^{48}$ Y. Rinne, J. Moen, K. Oksavik, and H. C. Carlson, J. Geophys. Res.: Space Phys. 112, A10313, https://doi.org/10.1029/2007JA012366 (2007).

${ }^{49}$ J. Moen, Y. Rinne, H. C. Carlson, K. Oksavik, R. Fujii, and H. Opgenoorth, J. Geophys. Res.: Space Phys. 113, A09220, https://doi.org/ 10.1029/2008JA013061 (2008).

${ }^{50}$ K. Oksavik, J. I. Moen, E. H. Rekaa, H. C. Carlson, and M. Lester, J. Geophys. Res.: Space Phys. 116, A12303, https://doi.org/10.1029/2011JA016788 (2011).

${ }^{51}$ M. Farge, Annu. Rev. Fluid Mech. 24, 395 (1992).

${ }^{52}$ C. Torrence and G. P. Compo, Bull. Am. Meteorol. Soc. 79, 61 (1998).

${ }^{53}$ H. C. Carlson, K. Oksavik, J. Moen, A. P. van Eyken, and P. Guio, Geophys. Res. Lett. 29, 24-1, https://doi.org/10.1029/2001GL014087 (2002).

${ }^{54}$ T. A. Bekkeng, K. S. Jacobsen, J. K. Bekkeng, A. Pedersen, T. Lindem, J.P. Lebreton, and J. I. Moen, Meas. Sci. Technol. 21, 085903 (2010).

${ }^{55}$ K. S. Jacobsen, A. Pedersen, J. I. Moen, and T. A. Bekkeng, Meas. Sci. Technol. 21, 085902 (2010).

${ }^{56}$ I. V. Golovchanskaya, B. V. Kozelov, and I. V. Despirak, Geomagn. Aeron. 52, 474, https://doi.org/10.1134/S0016793212040044 (2012).

${ }^{57}$ M. A. Reynolds and G. Ganguli, Phys. Plasmas 5, 2504 (1998).

${ }^{58}$ P. Palmadesso, G. Ganguli, and Y. C. Lee, "A new mechanism for excitation of waves in a magnetoplasma II. Wave-particle and nonlinear aspects," in Ion Acceleration in the Magnetosphere and Ionosphere (American Geophysical Union, 1986), pp. 301-306. 
${ }^{59}$ K.-I. Nishikawa, G. Ganguli, Y. C. Lee, and P. J. Palmadesso, Phys. Fluids 31, 1568 (1988).

${ }^{60}$ K.-I. Nishikawa, G. Ganguli, Y. C. Lee, and P. J. Palmadesso, J. Geophys. Res.: Space Phys. 95, 1029, https://doi.org/10.1029/JA095iA02p01029 (1990).

${ }^{61}$ R. Bruno, B. Bavassano, E. Pietropaolo, V. Carbone, and P. Veltri, Geophys. Res. Lett. 26, 3185, https://doi.org/10.1029/1999GL010668 (1999).
${ }^{62}$ S. W. Y. Tam, T. Chang, P. M. Kintner, and E. Klatt, Geophys. Res. Lett. 32, 105109, https://doi.org/10.1029/2004GL021445 (2005).

${ }^{63}$ G. Consolini and P. De Michelis, Geophys. Res. Lett. 32, 105101, https:// doi.org/10.1029/2004GL022063 (2005).

${ }^{64}$ D. Lagoutte, J. C. Cerisier, J. L. Plagnaud, J. P. Villain, and B. Forget, J. Atmos. Terr. Phys. 54, 1283 (1992).

${ }^{65}$ M. Yamauchi and R. Lundin, Space Sci. Rev. 95, 457 (2001). 